\title{
A note on the penalty parameter in Nitsche's method for unfitted boundary value problems
}

\author{
Frits de Prenter ${ }^{\mathrm{a}, *}$, Christoph Lehrenfeld ${ }^{\mathrm{b}}$, André Massing $^{\mathrm{c}}$ \\ ${ }^{a}$ Department of Mechanical Engineering, Eindhoven University of Technology, The Netherlands \\ ${ }^{b}$ Institute for Numerical and Applied Mathematics, University of Göttingen, Germany \\ ${ }^{c}$ Department of Mathematics and Mathematical Statistics, Umeå University, Sweden
}

\begin{abstract}
Nitsche's method is a popular approach to implement Dirichlet-type boundary conditions in situations where a strong imposition is either inconvenient or simply not feasible. The method is widely applied in the context of unfitted finite element methods. From the classical (symmetric) Nitsche's method it is well-known that the stabilization parameter in the method has to be chosen sufficiently large to obtain unique solvability of discrete systems. In this short note we discuss an often used strategy to set the stabilization parameter and describe a possible problem that can arise from this. We show that in specific situations error bounds can deteriorate and give examples of computations where Nitsche's method yields large and even diverging discretization errors.
\end{abstract}

Keywords: Nitsche's method, unfitted/immersed finite element methods, penalty/stabilization parameter, accuracy, stability, error analysis

\section{Introduction}

We consider the discretization of an unfitted Poisson problem. The problem domain is described separately from the encapsulating mesh, on which a finite element basis is defined. We consider the restriction of this finite element space with respect to the problem domain. Such an approach is used in many methods which are similar in virtue, e.g., the fictitious domain method [1], the cut finite element method (CutFEM) 2 [5], the finite cell method (FCM) 6-12, immersogeometric analysis 13-15, the immersed boundary method [16, the unfitted discontinuous Galerkin method (UDG) [17, [1], the extended finite element method (XFEM) [19 24, and several others. To impose essential boundary conditions, many of these methods apply some version of the classical Nitsche's method [25].

This requires stabilization by a penalization parameter to preserve the coercivity of the bilinear operator. Without additional stabilization of cut elements with e.g., ghost penalty terms [2, 26] - which is customary for methods referred to as CutFEM - this penalty parameter depends on the shape and size of the cut elements, i.e., it depends on the position of the geometry relative to the computational mesh. A typical choice which is sufficient to provide unique solvability of discrete problems, is to choose the stabilization parameter as an element wise constant and proportional to the ratio between the surface measure of the intersection between an element and the boundary and the volume measure of the intersection of the same element and the domain. As this ratio can become arbitrarily large, the stabilization parameter is not generally bounded.

In the mathematical literature, e.g., [2, 3], unfitted Nitsche formulations are generally supplemented by the aforementioned additional stabilization to bound the stabilization parameter in order to prove properties

\footnotetext{
${ }^{*}$ Corresponding author. Tel.: +31 615162599

Email addresses: f.d.prenter@tue.nl (Frits de Prenter), lehrenfeld@math.uni-goettingen.de (Christoph Lehrenfeld), andre.massing@umu.se (André Massing)
} 
of the method. In our opinion, the importance of this has not sufficiently been addressed in the literature however. Moreover, the method has effectively (and successfully) been applied without additional stabilization in the engineering literature, see e.g., [10 12. With this note, we therefore detailedly treat the possible problem that can occur when no additional measures are taken to bound the stabilization parameter and aim to provide a disclaimer for directly applying the classical form of Nitsche's method to immersed problems. To this end, we discuss and analyze the method and demonstrate that it can lead to poor results in the discretization error when the geometry intersects the computational domain such that large values of the stabilization parameter are required. We also investigate different situations where degenerated geometry configurations yield satisfactory and unsatisfactory results and provide a possible interpretation. We further review alternative formulations and possible modifications and stabilizations of the method.

Section 2 of this note introduces a model problem, followed by the Nitsche's method under consideration in Section 3. In Section 4 we carry out a simple error analysis of the method in a norm which is natural to the formulation. This analysis reveals the possible large discretization errors for unfortunate cut configurations. In Section 5 we give examples of bad cut configurations and show how these can lead to large and even degenerating discretization errors. Finally, we list alternative approaches to impose boundary conditions and variants of the classical Nitsche's method to circumvent this possible problem in Section 6

\section{The model problem}

As a model for more general elliptic boundary value problems, we consider the Poisson problem with inhomogeneous Dirichlet boundary data posed on an open and bounded domain $\Omega \subset \mathbb{R}^{d}$ with Lipschitz boundary $\partial \Omega$ :

$$
\begin{aligned}
-\Delta u=f & \text { in } \Omega, \\
u=g & \text { on } \quad \partial \Omega .
\end{aligned}
$$

We assume that the properties of the domain $\Omega$ imply that a shift theorem of the form:

$$
\|u\|_{H^{2}(\Omega)} \lesssim\|f\|_{L^{2}(\Omega)}+\|g\|_{H^{\frac{1}{2}}(\partial \Omega)},
$$

holds whenever $f \in L^{2}(\Omega)$ and $g \in H^{\frac{1}{2}}(\partial \Omega)$, e.g., when $\partial \Omega$ is smooth or $\Omega \subset \mathbb{R}^{2}$ is a convex domain with piecewise $C^{2}$ boundary [27]. In this note $\lesssim$ is used to denote an inequality with a constant that is independent of the mesh. This problem has the standard well-posed weak formulation: Find $u \in H_{g}^{1}(\Omega)$, such that:

$$
\int_{\Omega} \nabla u \nabla v d x=\int_{\Omega} f v d x \text { for all } v \in H_{0}^{1}(\Omega)
$$

In this formulation $H_{0}^{1}(\Omega)$ and $H_{g}^{1}(\Omega)$ are the standard Sobolev spaces with corresponding homogeneous and inhomogeneous boundary data. For simplicity, we assume that Dirichlet boundary conditions are posed on the whole boundary of $\Omega$. However, the results presented in this work extend to the case where Dirichlet boundary data is only prescribed on a part of the boundary as demonstrated in Section 5 .

\section{A Nitsche-based unfitted finite element method}

We consider a (triangular or rectilinear) shape regular background discretization $\tilde{\mathcal{T}}$ which encapsulates the domain $\Omega$. To each element $T \in \widetilde{\mathcal{T}}$ we associate the local mesh size $h_{T}=\operatorname{diam}(T)$ and define the global mesh size by $h=\max _{T \in \widetilde{\mathcal{T}}} h_{T}$. We define the active mesh $\mathcal{T}$ by:

$$
\mathcal{T}=\{T \in \tilde{\mathcal{T}}: T \cap \Omega \neq \emptyset\}
$$

and set $\widetilde{\Omega}=\bigcup_{T \in \mathcal{T}} T$, denoting the union of all elements from the active mesh. The set of geometric entities are illustrated in Figure 1. On the active mesh $\mathcal{T}$ we define the finite dimensional function space: 


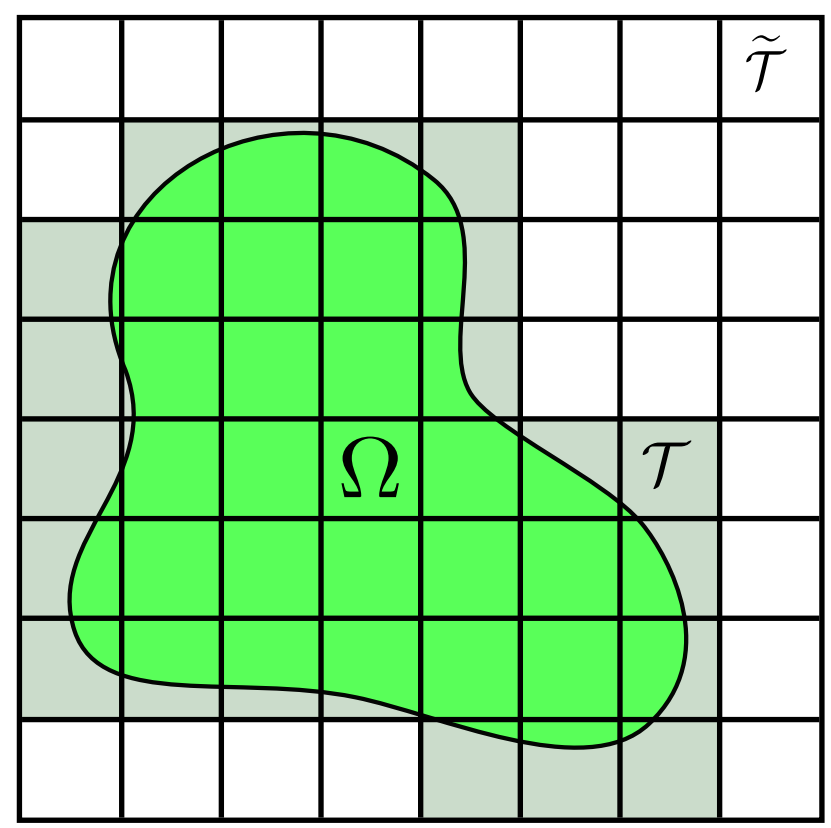

Figure 1: Example of a domain $\Omega$ with background mesh $\widetilde{\mathcal{T}}$ and active mesh $\mathcal{T}$.

$$
V_{h}=\left\{v \in C(\widetilde{\Omega}):\left.v\right|_{T} \in P_{k}(T), T \in \mathcal{T}\right\},
$$

consisting of continuous piecewise polynomials of order $k$. A simple prototype formulation for a Nitschebased unfitted finite element method is to find $u_{h} \in V_{h}$, such that:

$$
a_{h}\left(u_{h}, v_{h}\right)=l_{h}\left(v_{h}\right) \quad \forall v_{h} \in V_{h},
$$

where:

$$
\begin{aligned}
a_{h}\left(u_{h}, v_{h}\right) & :=\int_{\Omega} \nabla u_{h} \nabla v_{h} \mathrm{~d} x+\int_{\partial \Omega}\left(-\partial_{n} u_{h}\right) v_{h} \mathrm{~d} s+\int_{\partial \Omega}\left(-\partial_{n} v_{h}\right) u_{h} \mathrm{~d} s+\int_{\partial \Omega} \lambda_{T} u_{h} v_{h} \mathrm{~d} s, \\
l_{h}\left(v_{h}\right) & :=\int_{\Omega} f v_{h} \mathrm{~d} x+\int_{\partial \Omega}\left(-\partial_{n} v_{h}+\lambda_{T} v_{h}\right) g \mathrm{~d} s .
\end{aligned}
$$

Here, $\partial_{n}$ denotes the partial derivative in normal direction of the boundary of $\Omega$ and $\lambda_{T}$ denotes a elementwise stabilization parameter. The choice of the stabilization parameter is crucial. As the review of the error analysis in Section 3 reveals, the local stabilization parameter $\lambda_{T}$ has to be chosen sufficiently large in order to guarantee the discrete coercivity of the bilinear form $a_{h}(\cdot, \cdot)$. A common - see e.g., [10 12] - strategy is to solve the eigenvalue problem:

$$
\begin{aligned}
\text { Find }\left(u_{\mu}, \mu_{T}\right) & \left.\in V_{h}\right|_{T} ^{0} \times \mathbb{R}, \text { such that: } \\
\int_{\partial \Omega \cap T}\left(\partial_{n} u_{\mu}\right)\left(\partial_{n} v_{h}\right) d s & =\mu_{T} \int_{\Omega \cap T} \nabla u_{\mu} \nabla v_{h} d s \quad \text { for all }\left.v_{h} \in V_{h}\right|_{T} ^{0},
\end{aligned}
$$

on each element $T$ which has a non-empty intersection with $\partial \Omega$, see for instance $\left[28\right.$. Here, $\left.V_{h}\right|_{T} ^{0}$ is the space of functions in $\left.V_{h}\right|_{T}$ that are $L^{2}(T)$-orthogonal to constants. Let $N_{T}=\operatorname{dim}\left(\left.V_{h}\right|_{T}\right)$, then (9) is an $\left(N_{T}-1\right)$-dimensional eigenvalue problem with only non-negative eigenvalues. Motivated by the forthcoming stability analysis, a suitable choice of the stabilization parameter is to set:

$$
\lambda_{T}=2 \max \left\{\mu_{T}\right\}=2 \max _{\left.u_{h} \in V_{h}\right|_{T} ^{0}} \frac{\int_{\partial \Omega \cap T}\left(\partial_{h} u_{h}\right)^{2} d s}{\int_{\Omega \cap T}\left|\nabla u_{h}\right|^{2} d x}>0 .
$$




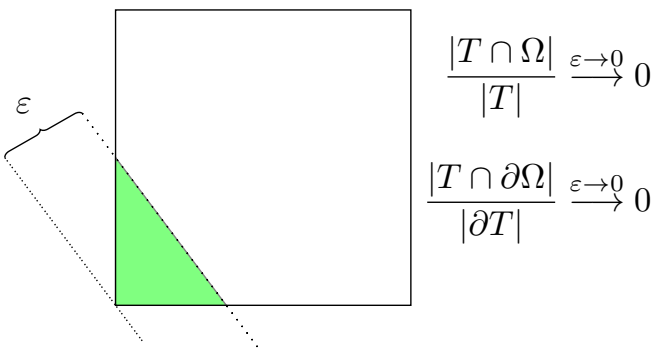

(a)

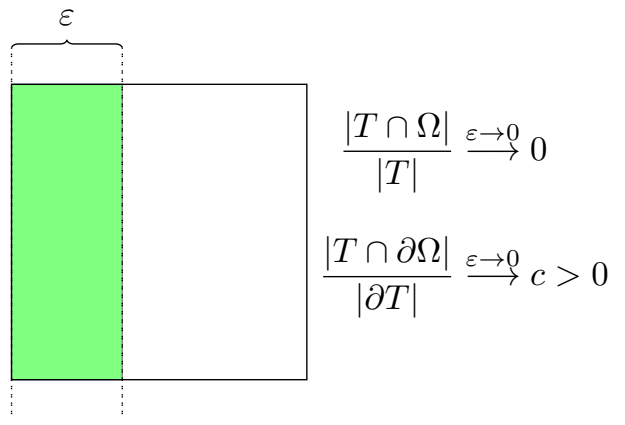

(b)

Figure 2: Different cut configurations. In (a) the part of the boundary in the element vanishes for vanishing volume, while for the sliver case (b) the measure of the boundary part stays bounded from below as the volume goes to zero.

Remark 1. With techniques as in [29], it can be shown that $\lambda_{T}$ scales with $|T \cap \partial \Omega|_{d-1} /|T \cap \Omega|_{d}$. Thus, shape-regularity implies that for a Nitsche-based formulation on a fitted and shape-regular mesh $\lambda_{T} \sim \mid T \cap$ $\left.\partial \Omega\right|_{d-1} /|T \cap \Omega|_{d} \sim h^{-1}$. In contrast, in an unfitted method, the ratio $|T \cap \partial \Omega|_{d-1} /|T \cap \Omega|_{d}$ depends not only on the cut element size but also highly on the cut configuration (see Figure 2). In particular, a sliver cut can lead to arbitrarily large values of $\lambda_{T}$ on relatively large parts of the boundary, depending on the thickness of the sliver. In Section 5 it is demonstrated how cut elements of approximately this shape can result in poor discretization errors.

Remark 2. To generate and solve the discrete linear system associated with problem (6) several issues have to be resolved, i.e., sufficiently accurate numerical integration on $T \cap \Omega$ and $T \cap \partial \Omega$ has to be provided, e.g., [30]39], and a particular tuning of the linear algebra solver might be necessary, e.g., [40, 41]. All these aspects are important, but in this work we focus only of the choice of $\lambda_{T}$ and its effect on the numerical solution, assuming that numerical integration can be performed sufficiently accurate and applying a direct solver to solve the linear systems exactly.

\section{A simple error analysis}

In this section we perform an error analysis for the variational form in (6). We first define a meshdependent norm in which a best approximation property holds in Section 4.1. In Section 4.2 we discuss the approximability in the mesh-dependent norm and the consequences for the discretization error in the $H^{1}(\Omega)$-norm.

\subsection{Best approximation in the mesh-dependent energy norm}

With the choice of the stabilization parameter as in $\sqrt{10}$, , the stability analysis is natural in the following mesh-dependent norm which we will refer to as the energy norm:

$$
\|v\|^{2}:=\|\nabla v\|_{\Omega}^{2}+\left\|\lambda_{T}^{-\frac{1}{2}} \partial_{n} v\right\|_{\partial \Omega}^{2}+\left\|\lambda_{T}^{\frac{1}{2}} v\right\|_{\partial \Omega}^{2}, \quad v \in V_{h} \oplus H^{2}(\Omega)
$$

We note that the use of this norm in the error analysis is essentially dictated by Nitsche's method and not by the model problem. We obtain coercivity and continuity in the following two lemmas.

Lemma 3 (Coercivity). There holds:

$$
a_{h}(u, u) \geq \frac{1}{5}\|u\|^{2} \text { for all } u \in V_{h} .
$$


Proof. Exploiting the eigenvalue characterization in the definition of $\lambda_{T}$ we obtain for arbitrary $\gamma>0$ :

$$
\begin{aligned}
a_{h}(u, u) & \geq\|\nabla u\|_{\Omega}^{2}+\left\|\lambda_{T}^{\frac{1}{2}} u\right\|_{\partial \Omega}^{2}-2 \int_{\partial \Omega}\left|\lambda_{T}^{-\frac{1}{2}} \partial_{n} u\right|\left|\lambda_{T}^{\frac{1}{2}} u\right| d s \\
& \geq\|\nabla u\|_{\Omega}^{2}+(1-\gamma)\left\|\lambda_{T}^{\frac{1}{2}} u\right\|_{\partial \Omega}^{2}-\frac{1}{\gamma}\left\|\lambda_{T}^{-\frac{1}{2}} \partial_{n} u\right\|_{\partial \Omega}^{2} \\
& \geq(1-\gamma)\|\nabla u\|_{\Omega}^{2}+(1-\gamma)\left\|\lambda_{T}^{\frac{1}{2}} u\right\|_{\partial \Omega}^{2}+\left(2 \gamma-\frac{1}{\gamma}\right)\left\|\lambda_{T}^{-\frac{1}{2}} \partial_{n} u\right\|_{\partial \Omega}^{2} \geq c\|u\|^{2},
\end{aligned}
$$

with $c=1-\gamma^{*}$ for $\gamma^{*}>0$ such that $1-\gamma^{*}=2 \gamma^{*}-\frac{1}{\gamma^{*}}$, which has only the positive solution $\gamma^{*}=\frac{1+\sqrt{13}}{6}$ so that $c>\frac{1}{5}$.

Lemma 4 (Continuity). There holds:

$$
a_{h}(u, v) \leq 2\|u\|\|v\| \text { for all } u, v \in V_{h} \oplus H^{2}(\Omega) .
$$

Proof. With Cauchy-Schwarz we directly obtain:

$$
\begin{aligned}
& a_{h}(u, v) \leq\|\nabla u\|_{\Omega}\|\nabla v\|_{\Omega}+\left\|\lambda_{T}^{-\frac{1}{2}} \partial_{n} u\right\|_{\partial \Omega}\left\|\lambda_{T}^{\frac{1}{2}} v\right\|_{\partial \Omega}+\left\|\lambda_{T}^{-\frac{1}{2}} \partial_{n} v\right\|_{\partial \Omega}\left\|\lambda_{T}^{\frac{1}{2}} u\right\|_{\partial \Omega}+\left\|\lambda_{T}^{\frac{1}{2}} u\right\|_{\partial \Omega}\left\|\lambda_{T}^{\frac{1}{2}} v\right\|_{\partial \Omega} \\
& \quad \leq\|\nabla u\|_{\Omega}\|\nabla v\|_{\Omega}+\left(\left\|\lambda_{T}^{-\frac{1}{2}} \partial_{n} u\right\|_{\partial \Omega}+\left\|\lambda_{T}^{\frac{1}{2}} u\right\|_{\partial \Omega}\right)\left(\left\|\lambda_{T}^{-\frac{1}{2}} \partial_{n} v\right\|_{\partial \Omega}+\left\|\lambda_{T}^{\frac{1}{2}} v\right\|_{\partial \Omega}\right) \\
& \quad \leq\left(\|\nabla u\|_{\Omega}^{2}+\left(\left\|\lambda_{T}^{-\frac{1}{2}} \partial_{n} u\right\|_{\partial \Omega}+\left\|\lambda_{T}^{\frac{1}{2}} u\right\|_{\partial \Omega}\right)^{2}\right)^{\frac{1}{2}}\left(\|\nabla v\|_{\Omega}^{2}+\left(\left\|\lambda_{T}^{-\frac{1}{2}} \partial_{n} v\right\|_{\partial \Omega}+\left\|\lambda_{T}^{\frac{1}{2}} v\right\|_{\partial \Omega}\right)^{2}\right)^{\frac{1}{2}} \\
& \quad \leq\left(\|\nabla u\|_{\Omega}^{2}+2\left\|\lambda_{T}^{-\frac{1}{2}} \partial_{n} u\right\|_{\partial \Omega}^{2}+2\left\|\lambda_{T}^{\frac{1}{2}} u\right\|_{\partial \Omega}^{2}\right)^{\frac{1}{2}}\left(\|\nabla v\|_{\Omega}^{2}+2\left\|\lambda_{T}^{-\frac{1}{2}} \partial_{n} v\right\|_{\partial \Omega}^{2}+2\left\|\lambda_{T}^{\frac{1}{2}} v\right\|_{\partial \Omega}^{2}\right)^{\frac{1}{2}} .
\end{aligned}
$$

Applying these lemmas and exploiting that the Nitsche formulation is consistent in the sense of Galerkin orthogonality, we obtain Céa's Lemma [42]:

Lemma 5 (Céa's Lemma). Let $u \in H^{2}(\Omega)$ be the solution to (1) and $u_{h} \in V_{h}$ be the solution to (6). Then there holds:

$$
\left\|u-u_{h}\right\| \leq 11 \inf _{v_{h} \in V_{h}}\left\|u-v_{h}\right\| .
$$

Proof. For an arbitrary $v_{h} \in V_{h}$ there holds $\left\|u-u_{h}\right\| \leq\left\|u-v_{h}\right\|+\left\|v_{h}-u_{h}\right\|$ and:

$$
\left\|v_{h}-u_{h}\right\|^{2} \leq 5 a_{h}\left(v_{h}-u_{h}, v_{h}-u_{h}\right)=5 a_{h}\left(v_{h}-u, v_{h}-u_{h}\right) \leq 10\left\|v_{h}-u_{h}\right\|\left\|v_{h}-u\right\|,
$$

s.t. the claim directly follows.

We note that in the proof we could not use coercivity for $u-u_{h}$, but only for discrete functions. To this end we applied the triangle inequality and were able to apply the coercivity estimate on the discrete function $v_{h}-u_{h}$, which was then combined with Galerkin orthogonality and continuity in the usual way.

This version of Céa's lemma suggests that Nitsche's method provides a robust and quasi-optimal method. The best approximation is, however, only provided in the mesh-dependent norm $\|\cdot\|$. In the next paragraph we discuss the discretization error of the best approximation in this norm.

\subsection{Approximability of the solution in the mesh-dependent energy norm}

We consider the approximability of the solution $u$ with the finite element space $V_{h}$ in the energy norm, i.e., $\inf _{v_{h} \in V_{h}}\left\|u-v_{h}\right\|$, which is the r.h.s. in (16). The aim of this paragraph is to show that the approximability in the discrete energy norm is not robust with respect to the position of the domain boundary. To this end, 
we split the energy norm, $c f$. (11), into two parts with one part that is robust and one part which is not robust:

$$
\inf _{v_{h} \in V_{h}}\left\|u-v_{h}\right\|^{2} \geq \inf _{v_{h} \in V_{h}}\left\{\left\|\nabla\left(u-v_{h}\right)\right\|_{\Omega}^{2}+\left\|\lambda_{T}^{-\frac{1}{2}} \partial_{n}\left(u-v_{h}\right)\right\|_{\partial \Omega}^{2}\right\}+\inf _{v_{h} \in V_{h}}\left\|\lambda_{T}^{\frac{1}{2}}\left(u-v_{h}\right)\right\|_{\partial \Omega}^{2} .
$$

We assume that the solution $u$ is smooth, $u \in H^{k+1}(\Omega)$, with $k \geq 1$ denoting the order of the discretization. In the sequel we use the notation $\gtrsim$ for an inequality with a constant that is independent of the cut configuration. For the first part of the r.h.s. in (18) we take the idea from [21] to show that the solution can be optimally approximated in $V_{h}$ with respect to these parts of the norm independent of the cut position. Afterwards, we show that the remaining part is the crucial problem which results in the missing robustness.

Let $\tilde{u} \in H^{k+1}(\widetilde{\Omega})$ be a continuous extension of $u$ to $\widetilde{\Omega}$ (the embedding domain, i.e., the union of all active elements), then we have:

$$
\left\|\nabla\left(u-v_{h}\right)\right\|_{\Omega}=\left\|\nabla\left(\tilde{u}-v_{h}\right)\right\|_{\Omega} \lesssim\left\|\nabla\left(\tilde{u}-v_{h}\right)\right\|_{\widetilde{\Omega}}, \quad \forall v_{h} \in V_{h} .
$$

To bound the latter part we can apply a standard best approximation result for $V_{h}$ on the fitted mesh $\widetilde{\mathcal{T}}$. This estimate is robust in the position of the boundary, i.e., there is a $v_{h} \in V_{h}$ s.t. $\left\|\nabla\left(u-v_{h}\right)\right\|_{\Omega} \lesssim$ $h^{k}\|\tilde{u}\|_{H^{k+1}(\widetilde{\Omega})} \lesssim h^{k}\|u\|_{H^{k+1}(\Omega)}$. Further, due to $\lambda_{T}^{-1} \lesssim h_{T}$ and a trace inequality, we have:

$$
\left\|\lambda_{T}^{-\frac{1}{2}} \partial_{n} v\right\|_{\partial \Omega \cap T} \lesssim\|v\|_{H^{1}(\Omega \cap T)}+h_{T}\|v\|_{H^{2}(\Omega \cap T)}, \quad \forall v \in H^{2}(\Omega \cap T) .
$$

Applying this to $u-v_{h}$ gives similar results (independent of the position of the boundary), hence $\| \lambda_{T}^{-\frac{1}{2}} \partial_{n}(u-$ $\left.v_{h}\right)\left\|_{\partial} \Omega \lesssim h^{k}\right\| u \|_{H^{k+1}(\Omega)}$.

We now consider the second term in 180 which is the crucial problem for the approximation of $u$ in the energy norm:

$$
\left\|\lambda_{T}^{\frac{1}{2}}\left(u-v_{h}\right)\right\|_{\partial \Omega}^{2}=\sum_{T \in \mathcal{T}} \lambda_{T}\left\|u-v_{h}\right\|_{T \cap \partial \Omega}^{2} .
$$

Let $h_{\Omega \cap T}:=\operatorname{diam}(\Omega \cap T)$ and $h_{\partial \Omega \cap T}:=\operatorname{diam}(\partial \Omega \cap T)$ be the characteristic lengths of the cut elements and their parts on the boundary. Furthermore, let $\tilde{v}_{h}$ be the best approximation of $u$ in $V_{h}$ in the energy norm. We have that $\left\|u-\tilde{v}_{h}\right\|_{T \cap \partial \Omega}^{2}$ is typically not zero and scales with $h_{\partial \Omega \cap T}^{2 k+2+(d-1)}, d-1$ denoting the dimension of the boundary. In the good case, if the cut element $T \cap \Omega$ is shape regular, there holds $h_{\partial \Omega \cap T} \sim h_{\Omega \cap T} \sim \lambda_{T}^{-1}$ such that we would have $\lambda_{T}\left\|u-\tilde{v}_{h}\right\|_{T \cap \partial \Omega}^{2} \leq c h_{\partial \Omega \cap T}^{2 k+1+(d-1)} \leq c h_{T}^{2 k+d}$ for some (bounded) constant $c>0$ which is a robust bound for the element contribution of $T$. In the bad case, if the cut element is not shape regular however, such as for a sliver cut element in Figure 2 it can occur that $\lambda_{T} \gg h_{\partial \Omega \cap T}^{-1}$, such that the contribution of a single element $\lambda_{T}\left\|u-\tilde{v}_{h}\right\|_{T \cap \partial \Omega}^{2}$ becomes unbounded.

We conclude that the last term in $(18)$ is unbounded and thus the same holds for the left hand side. Therefore the discretization error in the mesh-dependent energy norm, regardless of the best approximation property, can not be bounded from above. This missing robustness is essentially due to the possible existence of unfortunate cut configurations such as sliver cuts, which introduce values of the penalization parameter, $\lambda_{T}$, that are not bounded in terms of the reciprocal of the length of the boundary intersecting element $T$, $h_{\partial \Omega \cap T}$.

The energy norm is very natural with respect to the (symmetric) Nitsche formulation. Nevertheless, one is typically not interested in this norm but rather in the $H^{1}(\Omega)$-norm of the error. To obtain a priori estimates for the error in the $H^{1}(\Omega)$-norm we use a Poincaré-type inequality and combine it with the result in Céa's lemma:

$$
\left\|u-u_{h}\right\|_{H^{1}(\Omega)} \lesssim\left\|u-u_{h}\right\| \lesssim \inf _{v_{h} \in V_{h}}\left\|u-v_{h}\right\| .
$$

Whilst the approximability in the energy norm is not robust with respect to the boundary position, these inequalities are independent of the boundary position Therefore we can not give an optimal a priori error estimate for the $H^{1}(\Omega)$-norm.

\footnotetext{
${ }^{\dagger}$ By e.g., Lemma B.63 in [43] we have $\|u\|^{2} \geq\|\nabla u\|_{\Omega}^{2}+\left\|\lambda_{T}^{\frac{1}{2}} u\right\|_{\partial \Omega}^{2} \geq\|\nabla u\|_{\Omega}^{2}+\tilde{\lambda}\|u\|_{\partial \Omega}^{2} \geq \delta^{2}\|v\|_{H^{1}(\Omega)}^{2}$, with $\tilde{\lambda} \sim 1 / h$ the lower bound for $\lambda_{T}$.
} 
Besides showing that the traditional method to show optimality does not hold for Nitsche's method, we can also give an intuitive derivation of why Nitche's method can yield bad approximations in the $H^{1}(\Omega)$ norm. To do this, we compare the energy and Sobolev norms. At first sight these norms are not even equivalent, as $\left\|\lambda^{-\frac{1}{2}} \partial_{n} v\right\|_{\partial \Omega}$ is not bounded by the $H^{1}(\Omega)$-norm. This holds for all (even unsymmetric or fitted) Nitsche-type formulations however, and is not related to the problem of unbounded stabilization parameters we are targeting here. When we restrict ourselves to the space $W_{h}=V_{h} \oplus\langle u\rangle$, both norms are equivalent:

$$
c\left\|v_{h}\right\|_{H^{1}(\Omega)} \leq\left\|v_{h}\right\| \leq C\left\|v_{h}\right\|_{H^{1}(\Omega)}, \quad \forall v_{h} \in W_{h} .
$$

To investigate the strength of this equivalence, we examine the constants $c$ and $C$ in 20 . The first constant $0<\oint^{\dagger}<c<1$ is bounded from below and above independent of $u$ or the cut configuration. The upper bound of $c$ follows from:

$$
c=\min _{v_{h} \in W_{h}} \frac{\left\|v_{h}\right\|}{\left\|v_{h}\right\|_{H^{1}(\Omega)}} \lesssim \min _{\left.v_{h} \in V_{h}\right|_{\partial \Omega} ^{\perp}} \frac{\left\|v_{h}\right\|}{\left\|v_{h}\right\|_{H^{1}(\Omega)}}=\min _{\left.v_{h} \in V_{h}\right|_{\partial \Omega} ^{\perp}} \frac{\left|v_{h}\right|_{H^{1}}}{\left\|v_{h}\right\|_{H^{1}(\Omega)}}<1,
$$

with $\left.V_{h}\right|_{\partial \Omega} ^{\perp}$ the restriction of $V_{h}$ to functions that do not intersect the boundary, i.e.,

$$
\left.V_{h}\right|_{\partial \Omega} ^{\perp}=\left\{v_{h} \in V_{h} \mid v_{h}=\partial_{n} v_{h}=0 \text { on } \partial \Omega\right\} .
$$

The second constant in $20, C$, does depend on the cut configuration:

$$
C=\max _{v_{h} \in W_{h}} \frac{\left\|v_{h}\right\|}{\left\|v_{h}\right\|_{H^{1}(\Omega)}} \geq \max _{v_{h} \in V_{h}} \frac{\left\|v_{h}\right\|}{\left\|v_{h}\right\|_{H^{1}(\Omega)}} .
$$

Due to the finite dimensionality of $W_{h}$ we have $C<\infty$, but, in contrast to $c, C$ clearly depends on $\lambda$ and therefore on the cut configuration. The inequality in (22) disregards the possibility that $\left\|\lambda_{T}^{-\frac{1}{2}} v_{h}\right\|_{\partial \Omega} \gg$ $\left\|v_{h}\right\|_{H^{1}(\Omega)}$ for $v_{h} \in W_{h}$ due to possibly large normal derivatives of solution $u$, i.e., the general limitation for Nitsche-type methods we mentioned before. But also when this is not the case, $C$ will be large for large values of $\lambda$ however. Therefore, depending on the cut configuration, the equivalence between the energy norm and the $H^{1}(\Omega)$-norm can degenerate, such that the projections in the different norms can deviate from each other. With the solution to Nitsche's method closely resembling the projection in the energy norm, this may deviate substantially from an optimal approximation in $H^{1}(\Omega)$.

Remark 6. Using $c$ and $C$ we can also extend (19):

$$
\left\|u_{h}-v_{h}\right\|_{H^{1}(\Omega)} \leq \frac{1}{c}\left\|u_{h}-v_{h}\right\| \leq 10 \frac{1}{c}\left\|u-v_{h}\right\| \leq 10 \frac{C}{c}\left\|u-v_{h}\right\|_{H^{1}(\Omega)}, \quad \forall v_{h} \in V_{h},
$$

such that:

$$
\left\|u-u_{h}\right\|_{H^{1}(\Omega)} \leq\left(10 \frac{C}{c}+1\right) \inf _{v_{h} \in V_{h}}\left\|u-v_{h}\right\|_{H^{1}(\Omega)} .
$$

This seems like a satisfying result, but note that $C / c \gg 1$ when $\lambda \gg 1$.

\section{Numerical examples}

In this section we will demonstrate some examples of situations where the previously discussed discretization leads to large values for the stabilization parameter. The aim of this section is to illustrate the problem described in Section 4 and to show that it is not just a problem in the a priori analysis, but really yields large discretization errors in practice.

The numerical integration on elements that only partially intersect the domain is performed using the bisection-based tessellation scheme proposed in [44] with a maximal refinement depth of two. This leads to an exact representation of the domain for the first three examples and a reasonable good geometric approximation for the other examples. To make sure the results of these last examples are not affected by geometrical errors, we adapt the boundary conditions to the approximated geometry. On the approximated geometry we apply Gauss quadrature such that functions of order $2 k+1$ are integrated exactly, in order to achieve an accurate integration of the force terms and the boundary conditions. 


\subsection{Example 1: An overlapping square on a triangular and quadrilateral mesh}

The simplest example of a discretization with sliver cuts as described in the Section 3 is the squared domain $\Omega=(-1-\varepsilon, 1+\varepsilon)^{2}$ with $0<\varepsilon \ll 1$ on a structured grid that aligns with $\Omega$ for $\varepsilon=0$. The domain and the grid are shown in Figure $3 \mathrm{a}$ (for the triangular mesh), in which it is clearly visible that the cut elements become of arbitrarily large aspect ratios for $\varepsilon \rightarrow 0$.

\section{Triangular grid}

We use a triangular mesh of isosceles right triangles with legs of length $h=1 / K$ with $K=16$ and continuous piecewise linear basis functions. We apply an element local stabilization parameter by the procedure described in Section 3 . We set up the problem such that the analytical solution equals $u=$ $\sin (\pi x)+\sin (\pi y)$ and impose Dirichlet conditions through the previously discussed Nitsche method on all boundaries.
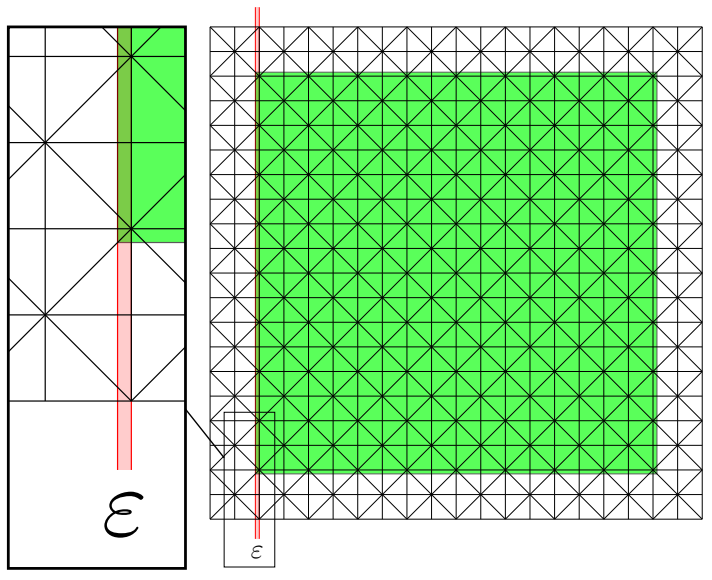

(a) Mesh and domain $\Omega$.

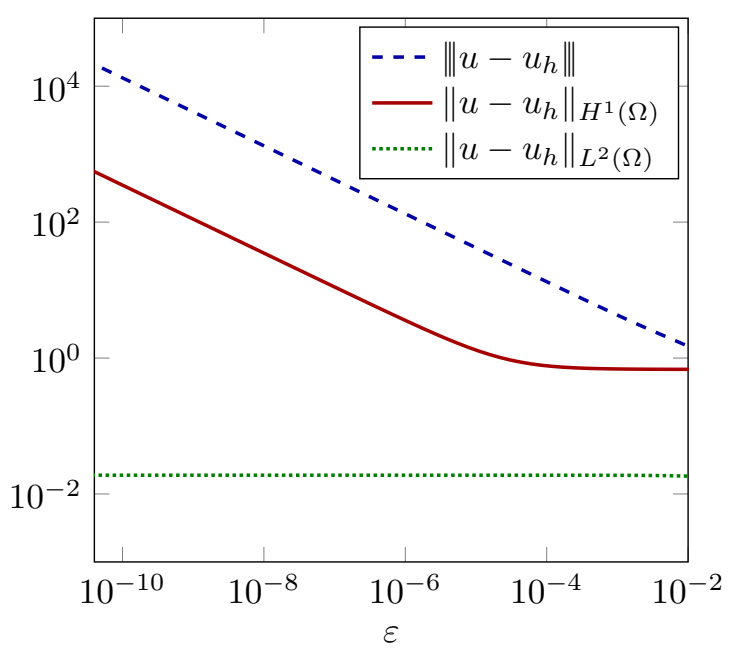

(b) Discretization errors.

Figure 3: Example 1 with a triangular grid.

The resulting discretization errors in the mesh-dependent energy norm, the $H^{1}(\Omega)$-norm and the $L^{2}(\Omega)$ norm are given in Figure $3 \mathrm{~b}$. From the results it is clearly visible that for $\varepsilon \rightarrow 0$ the discretization error degenerates in both the energy as the $H^{1}(\Omega)$-norm.

\section{Quadrilateral grid}

We repeat the example on a quadrilateral grid with the same nodal positions as the triangular grid, i.e., the mesh size $h=1 / K$ with $K=16$, see Figure $4 a$. Piecewise bilinear elements are used which lead to the same number of degrees of freedom. The results are shown in Figure 4b from which we can observe the same tendencies for the convergence $\varepsilon \rightarrow 0$.

We observe that the impact of a small $\varepsilon$ is less severe on the quadrilateral grid compared to the triangular grid. The error is several orders of magnitude smaller and the diverging effect only starts at a smaller value of $\varepsilon$. This difference is explained in the next paragraph, which is not a rigorous proof, but gives an intuitive illustration of the nature of the difference. It further serves as a motivation for the design of Examples 2 and 3 .

\section{Discussion of the difference between triangular and quadrilateral grids}

In Section 4.1 we have seen that the numerical solution closely approximates the energy norm projection of the analytical solution on the finite dimensional function space. For large values of $\lambda_{T}$, the contribution of normal derivatives to the energy norm diminishes and we can consider the energy norm to be a weighted combination of an $L^{2}(\partial \Omega)$-norm on the boundary and an $H_{0}^{1}(\Omega)$-seminorm in the interior. For increasing 

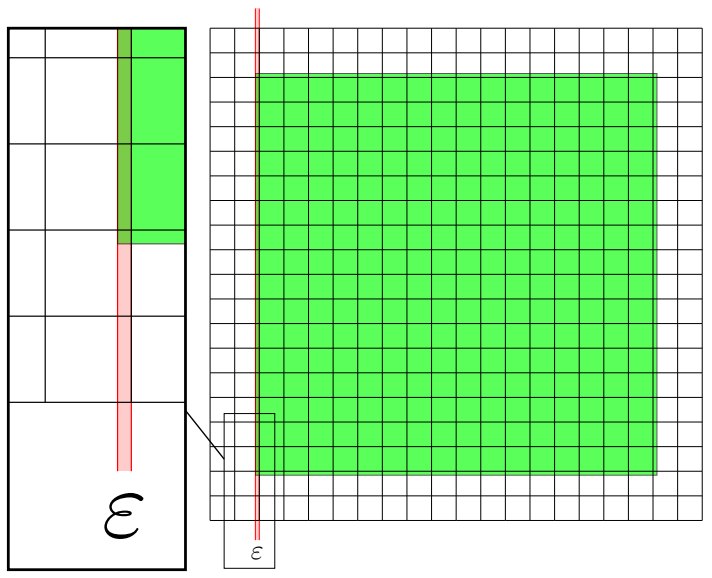

(a) Mesh and domain $\Omega$.

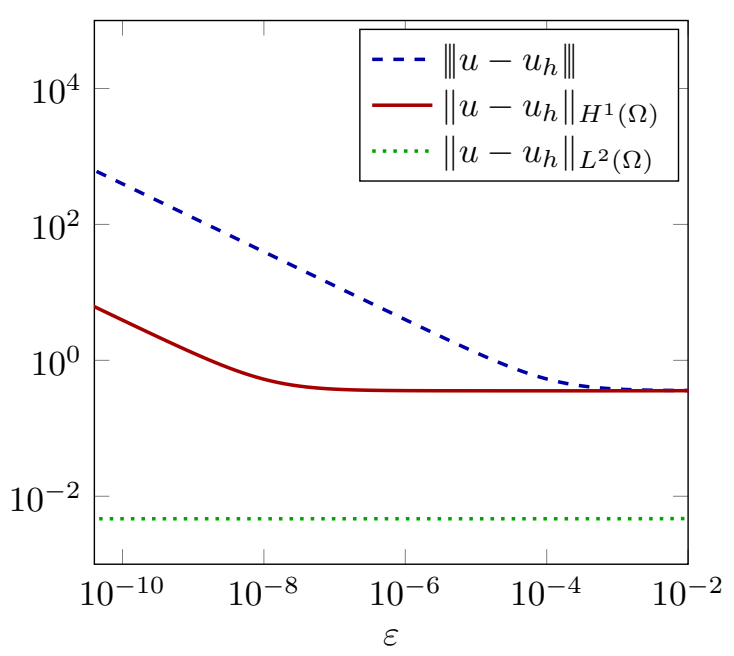

(b) Discretization errors.

Figure 4: Example 1 with a quadrilateral grid.

$\lambda_{T} \propto \varepsilon^{-1}$, the balance between these weighted norms is lost, and the energy norm projection basically consists of an $L^{2}(\partial \Omega)$-norm projection for all d.o.f.'s supported on the boundary and an $H_{0}^{1}(\Omega)$-seminorm projection in the interior for the remaining d.o.f.'s. Hence, in the limit of $\varepsilon \rightarrow 0$, the numerical solution $u_{h}$ satisfies:

$$
\left.u_{h}\right|_{\partial \Omega} \stackrel{\varepsilon \rightarrow 0}{\longrightarrow} u_{h}^{*}:=\underset{\left.v_{h} \in V_{h}\right|_{\partial \Omega}}{\operatorname{argmin}}\left\|\lambda_{T}^{\frac{1}{2}}\left(v_{h}-g\right)\right\|_{L^{2}(\partial \Omega)}^{2} .
$$

Hence $M:=\operatorname{dim}\left(\left.V_{h}\right|_{\partial \Omega}\right)$ d.o.f.'s are fixed independently of the remaining part of the variational formulation. Note that $M=16 K+7$ for the triangular grid (corresponding to the number of dots minus one in Figure 5a) and that $M=8 K+8$ for the quadrilateral grid (corresponding directly to the number of dots in Figure 5b). Besides constraining the boundary values, the $L^{2}(\partial \Omega)$-norm projection on the boundary can also affect the normal derivatives or even the complete numerical solution in the cut elements however. Let $N$ denote the number of basis functions with support on $\partial \Omega$ (squares in Figure 5), then we have $N=16 K+8$ for both meshes. Note that $N$ is also equal to the number of basis functions supported on cut elements. For the triangular grid we have $M=N-1$, which implies that in the limit of $\varepsilon \rightarrow 0$ only one d.o.f. in all cut elements together is not constrained by the boundary condition. For the quadrilateral mesh we have $M=1 / 2 N+4$, such that only approximately half of the d.o.f.'s supported on the boundary is constrained by the boundary condition. Along the straight parts of the boundary, the normal derivatives can therefore be set by the $H_{0}^{1}(\Omega)$-seminorm projection in the interior independent of the boundary condition. Only in the corners the normal derivatives are constrained by the boundary condition. As the problematic area is much smaller compared to the case with the triangular mesh, the impact of large stabilization parameters is - although asymptotically the same - less severe.

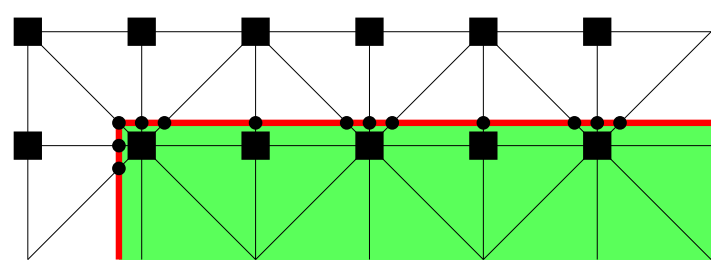

(a) Triangular grid.

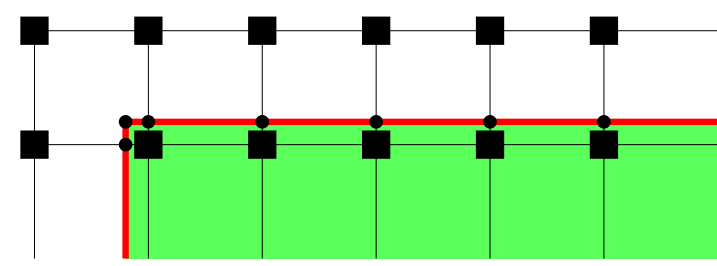

(b) Quadrilateral grid.

Figure 5: Sketch of d.o.f.'s of $V_{h}$ (squares) and d.o.f.'s of $\left.V_{h}\right|_{\partial \Omega}$ (dots).

From Figures $3 \mathrm{~b}$ and $4 \mathrm{~b}$ it is clear that small values of $\varepsilon$ do not only yield bad approximations, but actually cause diverging discretization errors. To explain this effect, we note that the boundary data $g$ has 
magnitudes $\sim 1$ whilst the basis functions that are only supported on cut elements have values of magnitude $\sim \varepsilon$ on the boundary. The $L^{2}(\partial \Omega)$-norm projection of the boundary condition therefore constrains the coefficients of these basis functions to a value of magnitude $\sim \varepsilon^{-1}$. This results in gradients in the cut elements that scale with $\varepsilon^{-1}$, which has also been observed by peak stresses in immersed elasticity problems 45, 46. These large gradients introduce an $\mathcal{O}\left(\varepsilon^{-\frac{1}{2}}\right)$ error in the $H^{1}(\Omega)$-norm, i.e., the sum of the volumes of the cut elements has a magnitude of $\sim \varepsilon$, whilst the error in the gradient squared has a value of magnitude $\sim \varepsilon^{-2}$. We notice that the $L^{2}(\Omega)$-norm error seems to be unaffected by the sliver cuts.

\subsection{Example 2: An overlapping square with mixed boundary conditions}

In this example we reuse the quadrilateral mesh and try to remove the problematic corner regions. To this end, we pose Neumann conditions on the left and right boundaries, $\partial \Omega_{N}=\{|x|=1+\varepsilon\}$, and Dirichlet conditions on the lower and upper boundaries, $\partial \Omega_{D}=\{|y|=1+\varepsilon\}$. Our hypothesis is that this will yield good approximation results in the $H^{1}(\Omega)$-norm for the quadrilateral grid, because in that case an $L^{2}\left(\partial \Omega_{D}\right)$-norm projection on the Dirichlet boundary will constrain $M=4 K+6$ d.o.f.'s whilst the number of basis functions supported on the Dirichlet boundary equals $N=2 M$. On all elements, including the corner elements, there is exactly one constraint for every two degrees of freedom. This allows to approximate the boundary values without affecting the normal derivatives.

The results are visible in Figure 6 and indeed show that in this situation the error in the $H^{1}(\Omega)$-norm is robust with respect to $\varepsilon$, even though the error in the mesh-dependent energy norm still scales with $\varepsilon^{-\frac{1}{2}}$. This is because also the best possible approximation of the boundary conditions has an error, such that the energy norm error increases for increasing $\lambda$. Furthermore, we notice that the errors in the energy norm are reduced by approximately a factor $\sqrt{2}$, which is accounted for by the reduction of the size of the Dirichlet boundary by a factor 2 .

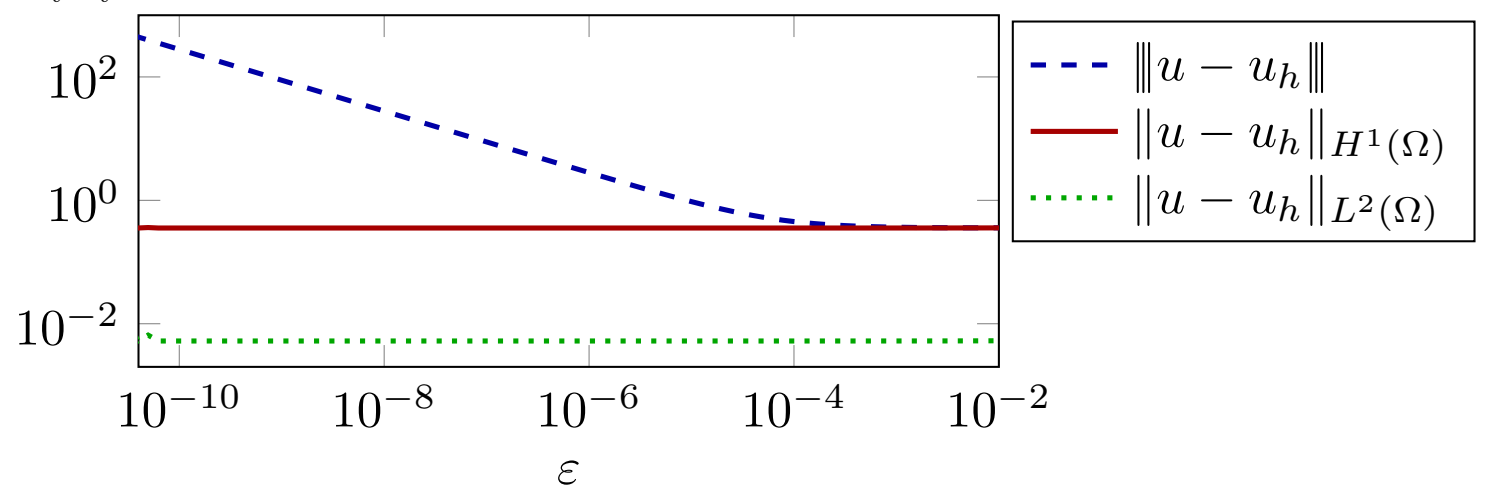

Figure 6: Discretization errors for Example 2.

\subsection{Example 3: A tilted square with mixed boundary conditions}

In this example we demonstrate that Example 2, be it useful for academic purposes, does not represent a general case for straight boundaries and tensor product grids. The reason that the second example yields robust approximation errors is that the Dirichlet boundary is parallel to a grid line, such that $N=2 M$, i.e., the number of d.o.f.'s constrained by the $L^{2}\left(\partial \Omega_{D}\right)$-norm projection is exactly right for linear bases. This is not generally the case for immersed methods however. In this third example we do not consider the square with boundaries $\partial \Omega_{N}=\{|x|=1+\varepsilon\}$ and $\partial \Omega_{D}=\{|y|=1+\varepsilon\}$, but consider a kinked domain with boundaries $\partial \Omega_{N}=\{|x-\varepsilon y|=1\}$ and $\partial \Omega_{D}=\{|y-\varepsilon x|=1\}$ as can be seen in Figure 7a. The (quadrilateral) grid and the analytical solution are the same as in the previous examples.

In this configuration we have $M=8 K+6$ constraints in the $L^{2}\left(\partial \Omega_{D}\right)$-norm projection on the Dirichlet boundary and $N=8 K+10$ basis functions that are supported on the Dirichlet boundary. Even though there is a kernel of dimension 4 in the projection of the boundary conditions on the nodes that are supported on the Dirichlet boundary, basis functions with values of magnitude $\sim \epsilon$ on the boundary will be constrained 


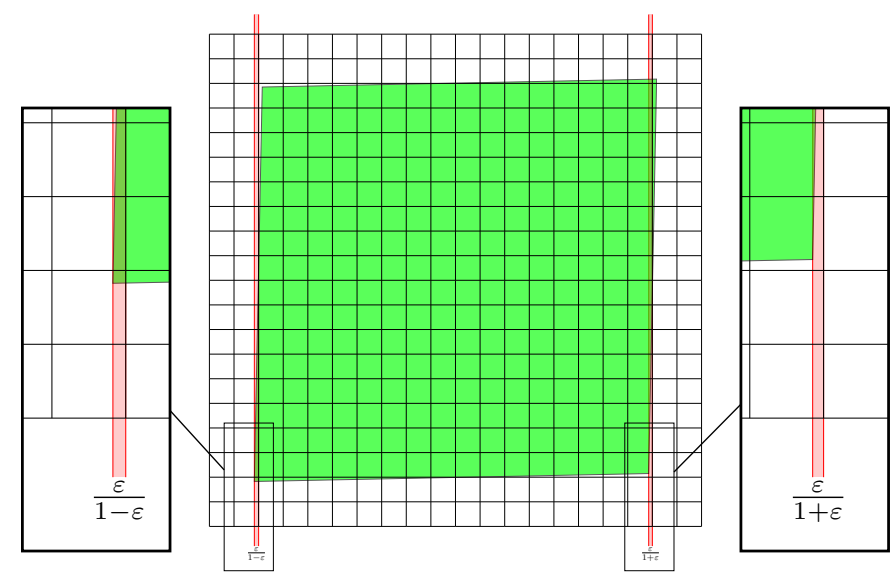

(a) Mesh and domain $\Omega$.

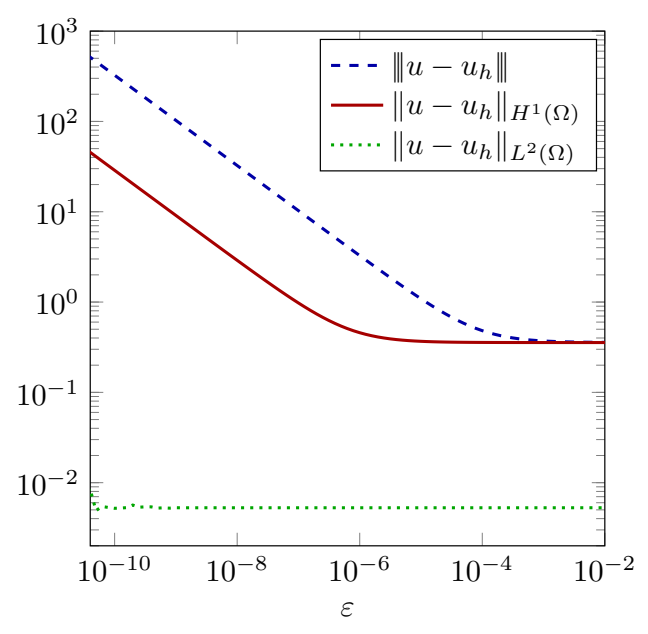

(b) Discretization errors.

Figure 7: Example 3.

to nodal values of magnitude $\sim \varepsilon^{-1}$. The results in Figure $7 \mathrm{~b}$ indeed show similar behavior to the first example.

\subsection{Example 4: A rounded square $\left(\|\cdot\|_{8}\right.$-norm unit ball)}

The previous examples consisted of domains with piecewise straight boundaries. Because unfitted methods are generally used for complex domains, this example involves a curved boundary that creates sliver-like cuts which can occur in many realistic applications. The boundary of the domain is defined by the function $x^{8}+y^{8}=(1+\varepsilon)^{8}$ and Dirichlet conditions are imposed on the complete boundary. A sketch of the domain is visible in Figure $8 \mathrm{a}$

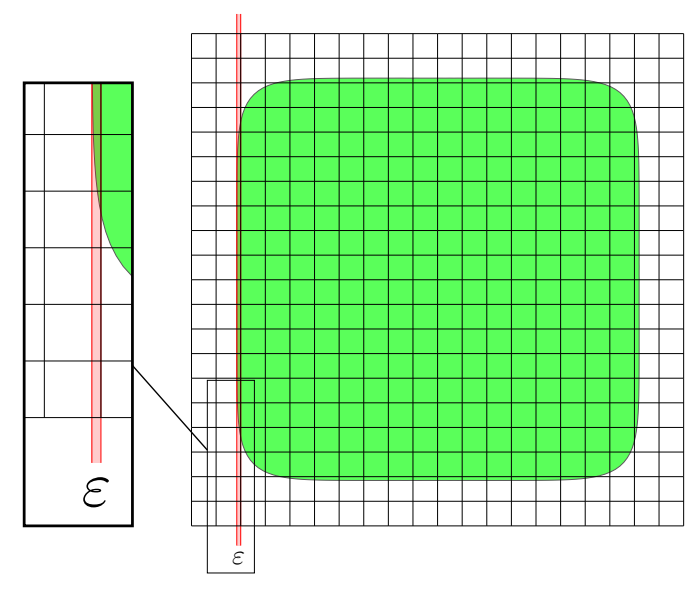

(a) Mesh and domain $\Omega$.

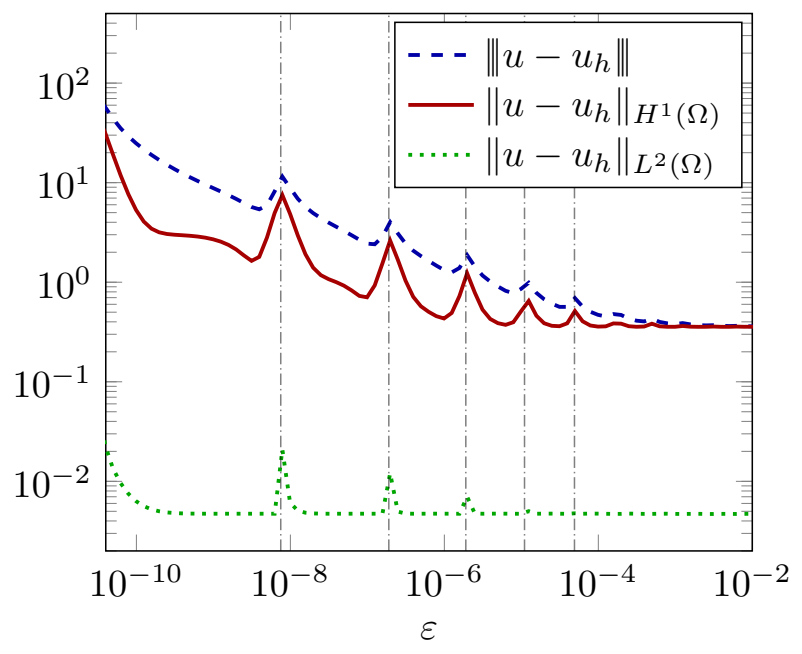

(b) Discretization errors.

Figure 8: Example 4.

The results are visible in Figure $8 \mathrm{~b}$. From the results we note that for this example not even the error in the $L^{2}(\Omega)$-norm is robust with respect to the cut configuration. Furthermore, a peculiar effect is observed as the errors don't simply increase for decreasing $\varepsilon$, but peak at certain values. This occurs because a smaller value of $\varepsilon$ doesn't simply imply thinner cut elements for this test case. The zones of the domain that constitute the thin (or sliver) cut elements - the parts of the domain for which $|x|>1$ or $|y|>1-$ do 
not only get thinner but also get shorter for decreasing $\varepsilon$. The intersections between the domain boundary and the grid lines $x= \pm 1$ and $y= \pm 1$ form the boundaries of these zones, and move towards the points $x= \pm 1, y=0$ and $x=0, y= \pm 1$ for $\varepsilon \rightarrow 0$. As a result, the cut elements become thinner for decreasing $\varepsilon$ until these intersections shift through a vertex, removing the thinnest cut element from the system. At this point the error decreases, after which it again increases until the intersection shifts through the next vertex. Counting the number of degrees of freedom in the system confirms that the errors peak at values of $\varepsilon$ where the intersection between the boundary and the grid lines $x= \pm 1$ and $y= \pm 1$ shift through a vertex, because at these values also the number of degrees of freedom is reduced, which is indicated by the vertical gray dash dotted lines in the figure.

\subsection{Higher order basis functions and three-dimensional domains}

The same effects as observed in the previous examples occur for higher order basis functions and threedimensional domains. The fourth example has also been used to investigate the behavior of a second order discretization. The results are visible in Figure 9 Note that this also requires a larger stabilization parameter as the space $\left.V_{h}\right|_{T} ^{0}$ in $(10)$ is larger for higher order bases. In Figure 10 the results of example 4 in a threedimensional setting are visible. The domain is defined as $x^{8}+y^{8}+z^{8}<(1+\varepsilon)^{8}$, Dirichlet conditions are imposed on the complete boundary and the approximated solution equals $u=\sin (\pi x)+\sin (\pi y)+\sin (\pi z)$. For this three-dimensional computation we have used a first order trilinear basis on a grid with size $h=1 / 8$ instead of $h=1 / 16$ and the maximal refinement depth has been reduced to 0 to save computation time. Also in three dimensions the same effect is observed.

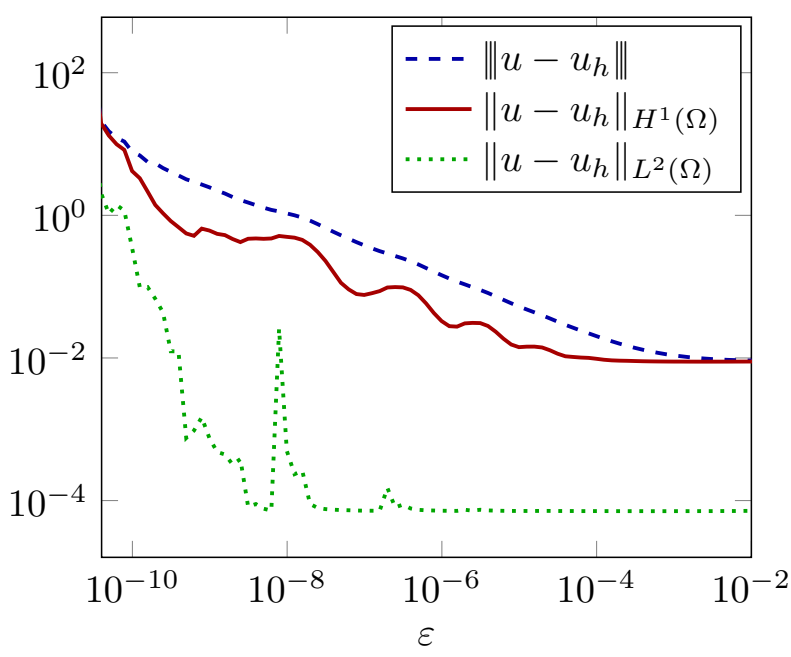

Figure 9: Second order B-splines.

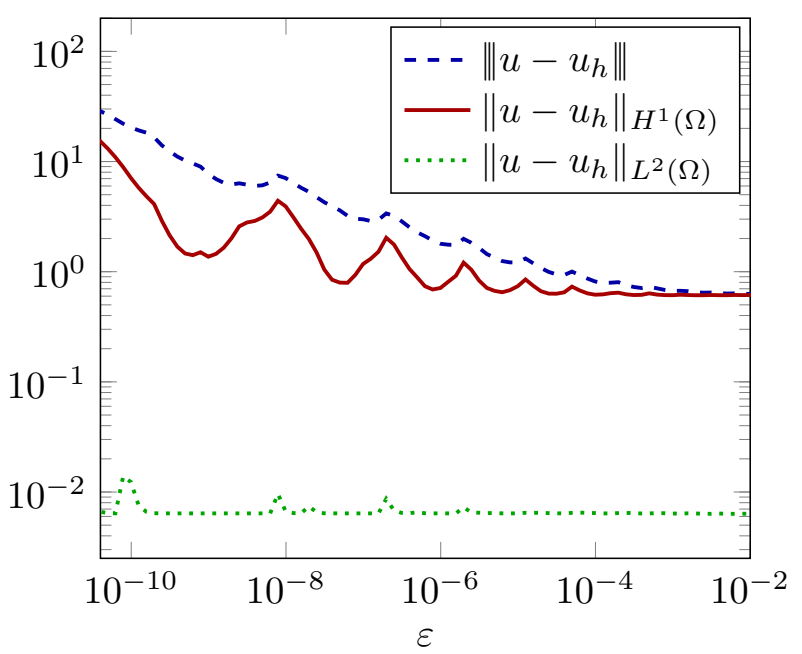

Figure 10: Three-dimensional example.

\subsection{Summary of observations}

In the previous examples we observed that sliver-like cut configurations (on Dirichlet boundaries) can cause severe problems in the discretization. The discretization error measured in the $H^{1}(\Omega)$-norm and the mesh-dependent energy norm can not be bounded independently of $\varepsilon$, the parameter that controls the volume ratios in the sliver cut elements. While the $L^{2}(\Omega)$-norm error seems to be robust for the simple cases, it is not for the more complex examples.

We tried to give an interpretation of the origins that trigger the large errors for small $\varepsilon$ and were able to characterize a difference in the relative number of d.o.f.'s involved in the projection of the Dirichlet conditions by the penalty terms. As a result, in Example 1 the effect is more severe for the triangular discretization than for the quadrilateral discretization. On specific domains, the instabilities due to the sliver cases are even not triggered at all, as demonstrated in Example 2. This is similar to penalty methods for boundary fitting discretizations, where exactly the right number of d.o.f.'s is involved in the penalty, such that very large penalties can be applied [47. However, small deviations immediately display the missing robustness 
with respect to $\varepsilon$ and lead to large errors, $c f$. Example 3. We have obtained similar results for a more complicated domain with curved boundaries, for quadratic basis functions and in three dimensions, such that the results do not only apply for piecewise (bi-)linear discretizations and two-dimensional problems on simple domains.

\section{Conclusion and resolutions of the problem}

From Section 4 and 5 it is evident that the stabilization parameter needs to be bounded in order to guarantee optimal approximation properties. In fact, several modifications of Nitsche's method that preclude the previously discussed problems already exist in the literature. We divide these in three groups. Firstly, we discuss alternatives to the symmetric Nitsche formulation. Secondly, there exist approaches to preclude degrees of freedom that have very small supports in the problem domain, such that the required stabilization parameter is bounded. Thirdly, we discuss a stabilization mechanism called the ghost penalty, which achieves a similar effect by penalizing jumps in normal derivatives along boundaries of cut elements.

\subsection{Alternative formulations}

The most obvious alternative for the symmetric Nitsche formulation is the unsymmetric Nitsche formulation, e.g., [48 50], which however is not adjoint consistent. Other methods to weakly impose Dirichlet conditions are the penalty method and the use of Lagrange multipliers and related methods, e.g., [47, 51,53. Another approach with unfitted grids is to manipulate the function space such that it allows for the strong imposition of boundary conditions, i.e., 54 56.

\subsection{Precluding d.o.f.'s with small supports in the problem domain}

When the system does not contain functions with very small supports in the problem domain, it follows from (10) that the required stabilization parameter is reduced. This is an additional advantage of methods that eliminate functions with very small supports from the system, which were initially introduced in order to improve the conditioning of the system matrix. The simplest way to achieve this is by simply excluding these basis functions from the basis, e.g., [28, 44, 56, 57, which however may decrease the accuracy. In [54, 55, 58, 60, functions with small supports in the problem domain are constrained to geometrically nearby functions, which decreases the required stabilization parameter without compromising the accuracy. Another approach that precludes basis functions from having a very small stiffness (having the same effect as precluding functions with a very small support) is using a fictitious domain stiffness, e.g., [12, which is thoroughly analyzed in [61].

\subsection{Ghost penalty}

The ghost penalty, see [2, 26], is a consistent penalty term that can be added to the bilinear form and which is customary in methods referred to as CutFEM, see e.g., 3]. The ghost penalty introduces a penalty in the vicinity of the boundary that weakly bounds polynomials of neighboring elements to coincide. This is done by adding terms that penalize jumps in the normal derivatives along element interfaces or local projection type penalties [62]. This weakly enforces higher order continuity along element interfaces near the boundary, and therefore relates functions with small support in the boundary region to interior functions. Similar to strongly enforcing this higher order continuity by constraining basis functions with small supports to geometrically nearby functions, this effectively controls and bounds the stabilization parameter. Besides bounding the stabilization parameter, penalizing the jumps in the normal derivatives gives control over the gradients in cut elements and therefore impede gradients of magnitude $\sim \varepsilon^{-1}$, which we observed in the cut elements.

Remark 7. Inspired by the observations in the experiments, we also suggest a quick fix that is simple to implement. To alleviate the problem of unbounded stabilization parameters, we modify Nitsche's method to a hybrid Nitsche-penalty method. First, we manually set an upper bound for the stabilization parameter $C_{\lambda}>0$. We then use Nitsche's method on the elements where the required stabilization parameter (computed 
as in (10) is smaller than this upper bound and use a penalty method (i.e., no flux terms) with penalty parameter $C_{\lambda}$ on the elements where Nitsche's method would require a larger value than this manually set upper bound. Bounding the penalty parameter is easily motivated from an accuracy point of view, when considering the results and analysis previously presented in this manuscript. It is noted that a pure penalty method is not consistent with the initial problem (9). This inconsistency can be argued to be acceptable however, considering that this generally only occurs on very small parts of the domain and that on these parts of the boundary the flux terms are negligibly small compared to the penalty terms anyway.

This hybrid Nitsche-penalty method yields good approximations in the $H^{1}(\Omega)$-norm for the fourth test case, with $C_{\lambda}=16 \cdot 10^{3}$. The results are visible in Figure 11 and show that the quality of the approximation is virtually independent of $\varepsilon$. This suggests that this could be a simple and straightforward fix for this problem. We consider this a preliminary result however, as this adaptation of the method has not undergone rigorous testing. Also setting the value of $C_{\lambda}$ needs a more thorough analysis and could require different values for different mesh sizes, higher order discretizations and other problems than Poisson's problem.

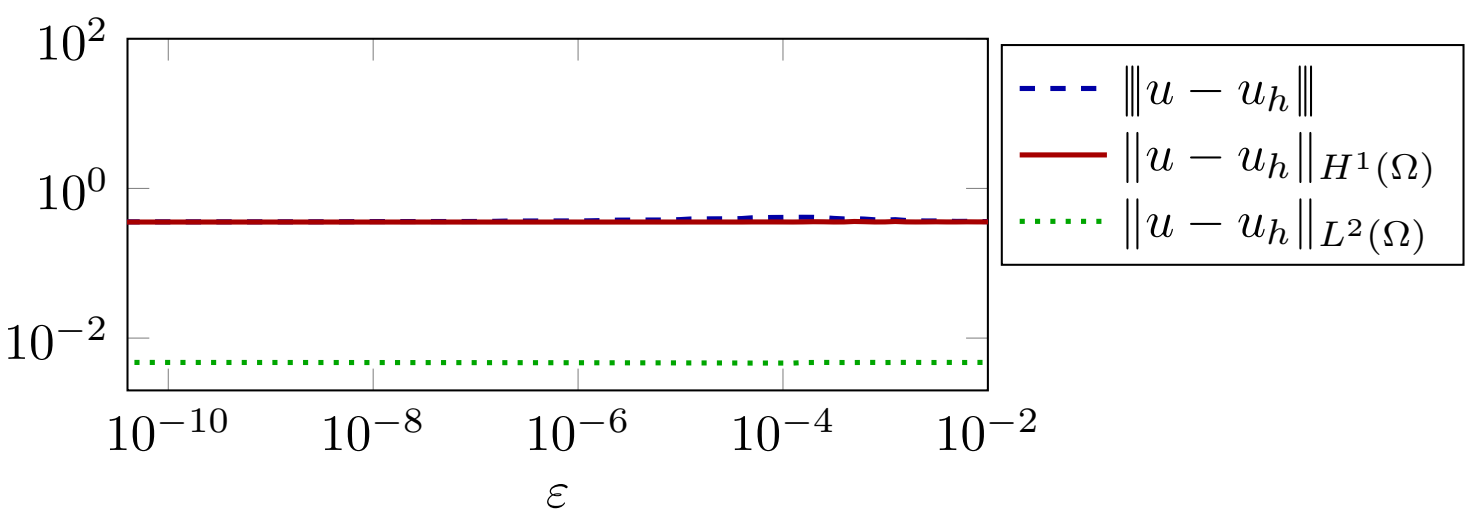

Figure 11: Discretization errors of the modification of Nitche's method for Example 4 . The energy norm error for this hybrid method is defined as: $\|v\|^{2}:=\|\nabla v\|_{\Omega}^{2}+\left\|\lambda_{T}^{-\frac{1}{2}} \partial_{n} v\right\|_{\Gamma^{\mathrm{Nitsche}}}^{2}+\left\|\lambda_{T}^{\frac{1}{2}} v\right\|_{\Gamma^{\mathrm{Nitsche}}}^{2}+\left\|C_{\lambda}^{\frac{1}{2}} v\right\|_{\Gamma^{\text {penalty }}}^{2}$.

\section{Acknowledgement}

The research of F. de Prenter was funded by the NWO under the Graduate Program Fluid \& Solid Mechanics. C. Lehrenfeld gratefully acknowledges funding by the German Science Foundation (DFG) within the project LE 3726/1-1.

\section{References}

[1] R. Glowinski, T. Pan, J. Periaux, A fictitious domain method for Dirichlet problem and applications, Computer Methods in Applied Mechanics and Engineering 111 (3-4) (1994) 283-303.

[2] E. Burman, P. Hansbo, Fictitious domain finite element methods using cut elements: II. A stabilized Nitsche method, Applied Numerical Mathematics 62 (4) (2012) 328-341.

[3] E. Burman, S. Claus, P. Hansbo, M. Larson, A. Massing, CutFEM: Discretizing geometry and partial differential equations, International Journal for Numerical Methods in Engineering 104 (7) (2014) 472-501.

[4] A. Massing, M. Larson, A. Logg, M. Rognes, A stabilized Nitsche fictitious domain method for the Stokes problem, Journal of Scientific Computing 61 (3) (2014) 604-628.

[5] E. Burman, S. Claus, A. Massing, A Stabilized Cut Finite Element Method for the Three Field Stokes Problem, SIAM Journal on Scientific Computing 37 (4) (2015) A1705-A1726.

[6] J. Parvizian, A. Düster, E. Rank, Finite cell method, Computational Mechanics 41 (1) (2007) 121-133.

[7] A. Düster, J. Parvizian, Z. Yang, E. Rank, The finite cell method for three-dimensional problems of solid mechanics, Computer Methods in Applied Mechanics and Engineering 197 (45) (2008) 3768-3782.

[8] D. Schillinger, L. Dede, M. Scott, J. Evans, M. Borden, E. Rank, T. Hughes, An isogeometric design-through-analysis methodology based on adaptive hierarchical refinement of NURBS, immersed boundary methods, and T-spline CAD surfaces, Computer Methods in Applied Mechanics and Engineering 249 (2012) 116-150. 
[9] E. Rank, M. Ruess, S. Kollmannsberger, D. Schillinger, A. Düster, Geometric modeling, isogeometric analysis and the finite cell method, Computer Methods in Applied Mechanics and Engineering 249 (2012) 104-115.

[10] M. Ruess, D. Schillinger, Y. Bazilevs, V. Varduhn, E. Rank, Weakly enforced essential boundary conditions for NURBSembedded and trimmed NURBS geometries on the basis of the finite cell methodod, International Journal for Numerical Methods in Engineering 95 (10) (2013) 811-846.

[11] M. Ruess, D. Schillinger, A. Özcan, E. Rank, Weak coupling for isogeometric analysis of non-matching and trimmed multi-patch geometries, Computer Methods in Applied Mechanics and Engineering 269 (2014) 46-71.

[12] D. Schillinger, M. Ruess, The Finite Cell Method: A review in the context of higher-order structural analysis of CAD and image-based geometric models, Archives of Computational Methods in Engineering (2015) 1-65.

[13] D. Kamensky, M.-C. Hsu, D. Schillinger, J. Evans, A. Aggarwal, Y. Bazilevs, M. Sacks, T. Hughes, An immersogeometric variational framework for fluid-structure interaction: Application to bioprosthetic heart valves, Computer Methods in Applied Mechanics and Engineering 284 (2015) 1005-1053.

[14] V. Varduh, M.-C. Hsu, M. Ruess, D. Schillinger, The tetrahedral finite cell method: Higher-order immersogeometric analysis on adaptive non-boundary-fitted meshes, International Journal for Numerical Methods in Engineering 107 (12) (2016) 1054-1079.

[15] F. Xu, D. Schillinger, D. Kamensky, V. Varduhn, C. Wang, M.-C. Hsu, The tetrahedral finite cell method for fluids: Immersogeometric analysis of turbulent flow around complex geometries, Computers \& Fluids 141 (2016) 135-154.

[16] C. Peskin, The immersed boundary method, Acta Numerica 11 (2002) 479-517.

[17] P. Bastian, C. Engwer, An unfitted finite element method using discontinuous Galerkin, International Journal for Numerical Methods in Engineering 79 (12) (2009) 1557-1576.

[18] R. Massjung, An Unfitted Discontinuous Galerkin Method Applied to Elliptic Interface Problems, SIAM Journal on Numerical Analysis 50 (6) (2012) 3134-3162.

[19] T. Belytschko, N. Moes, S. Usui, C. Parimi, Arbitrary discontinuities in finite elements, International Journal for Numerical Methods in Engineering 50 (4) (2001) 993-1013.

[20] T.-P. Fries, T. Belytschko, The extended/generalized finite element method: an overview of the method and its applications, International Journal for Numerical Methods in Engineering 84 (3) (2010) 253-304.

[21] A. Hansbo, P. Hansbo, An unfitted finite element method, based on Nitsche's method, for elliptic interface problems, Computer Methods in Applied Mechanics and Engineering 191 (47-48) (2002) 5537 - 5552.

[22] S. Groß, V. Reichelt, A. Reusken, A finite element based level set method for two-phase incompressible flows, Computing and Visualization in Science 9 (4) (2006) 239-257.

[23] R. Becker, E. Burman, P. Hansbo, A Nitsche extended finite element method for incompressible elasticity with discontinuous modulus of elasticity, Computer Methods in Applied Mechanics and Engineering 198 (41) (2009) 3352 - 3360.

[24] R. Becker, E. Burman, P. Hansbo, A hierarchical NXFEM for fictitious domain simulations, International Journal for Numerical Methods in Engineering 86 (4) (2011) 549-559.

[25] J. Nitsche, Über ein Variations zur Lösung von Dirichlet-Problemen bei Verwendung von Teilräumen die keinen Randbedingungen unterworfen sind, Abhandlungen aus dem mathematischen Seminar der Universität Hamburg 36 (1) (1971) 9-15.

[26] E. Burman, Ghost penalty, Comptes Rendus Mathematique 348 (21) (2010) 1217-1220.

[27] P. Grisvard, Elliptic problems in nonsmooth domains, Pitman, 1985.

[28] A. Embar, J. Dolbow, I. Harari, Imposing Dirichlet boundary conditions with Nitsche's method and spline based finite elements, International Journal for Numerical Methods in Engineering 83 (7) (2010) 877-898.

[29] T. Warburton, J. Hesthaven, On the constants in hp-finite element trace inverse inequalities, Computer Methods in Applied Mechanics and Engineering 192 (25) (2003) 2765-2773.

[30] Z. Yang, M. Ruess, S. Kollmannsberger, A. Düster, E. Rank, An efficient integration technique for the voxel-based finite cell method, International Journal for Numerical Methods in Engineering 91 (5) (2012) 457-471.

[31] A. Abedian, J. Parvizian, A. Düster, H. Khademyzadeh, E. Rank, Performance of different integration schemes in facing discontinuities in the finite cell method, International Journal of Computational Methods 10 (3).

[32] S. Duczek, U. Gabbert, Efficient integration method for fictitious domain approaches, Computational Mechanics 56 (4) (2015) 725-738.

[33] T.-P. Fries, S. Omerović, Higher-order accurate integration of implicit geometries, International Journal for Numerical Methods in Engineering 106 (5) (2015) 323-371.

[34] C. Lehrenfeld, High order unfitted finite element methods on level set domains using isoparametric mappings, Computer Methods in Applied Mechanics and Engineering 300 (2016) 716-733.

[35] M. Joulaian, S. Hubrich, A. Düster, Numerical integration of discontinuities on arbitrary domains based on moment fitting, Computational Mechanics 57 (6) (2016) 979-999.

[36] L. Kudela, N. Zander, S. Kollmannsberger, E. Rank, Smart octrees: Accurately integrating discontinuous functions in 3D, Computer Methods in Applied Mechanics and Engineering 306 (2016) 406-426.

[37] V. Thiagarajan, V. Shapiro, Adaptively Weighted Numerical Integration in the Finite Cell Method, Computer Methods in Applied Mechanics and Engineering 311 (2016) 250-279.

[38] A. Stavrev, L. Nguyen, R. Shen, V. Varduhn, M. Behr, S. Elgeti, D. Schillinger, Geometrically accurate, efficient, and flexible quadrature techniques for the tetrahedral finite cell method, Computer Methods in Applied Mechanics and Engineering 310 (2016) 646-673.

[39] T.-P. Fries, S. Omerović, D. Schöllhammer, J. Steidl, Higher-order meshing of implicit geometries - Part i: Integration and interpolation in cut elements, Computer Methods in Applied Mechanics and Engineering 313 (2017) $759-784$.

[40] C. Lehrenfeld, A. Reusken, Optimal preconditioners for Nitsche-XFEM discretizations of interface problems, Numerische 
Mathematik 135 (2) (2016) 313-332.

[41] F. de Prenter, C. Verhoosel, G. van Zwieten, E. van Brummelen, Condition number analysis and preconditioning for the finite cell method, Computer Methods in Applied Mechanics and Engineering 316 (2017) 297-327.

[42] J. Céa, Approximation variationnelle des problèmes aux limites, Ph.D. thesis (1964).

[43] A. Ern, J. Guermond, Theory and Practice of Finite Elements, Springer, 2004.

[44] C. Verhoosel, G. Van Zwieten, B. Van Rietbergen, R. De Borst, Image-based goal-oriented adaptive isogeometric analysis with application to the micro-mechanical modeling of trabecular bone, Computer Methods in Applied Mechanics and Engineering 284 (2015) 138-164.

[45] L. van Miegroet, P. Duysinx, Stress concentration minimization of 2D filets using X-FEM and level set description, Structural and Multidisciplinary Optimization 33 (4) (2007) 425-438.

[46] W. Jiang, C. Annavarapu, J. Dolbow, I. Harari, A robust Nitsche's formulation for interface problems with spline-based finite elements, International Journal for Numerical Methods in Engineering 104 (7) (2015) 676-696.

[47] I. Babuška, The finite element method with penalty, Mathematics of Computation 27 (122) (1973) 221-228.

[48] E. Burman, A penalty-free nonsymmetric Nitsche-type method for the weak imposition of boundary conditions, SIAM Journal on Numerical Analysis 50 (4) (2012) 1959-1981.

[49] T. Boiveau, E. Burman, A penalty-free Nitsche method for the weak imposition of boundary conditions in compressible and incompressible elasticity, IMA Journal of Numerical Analysis 36 (2) (2016) 770-795.

[50] D. Schillinger, I. Harari, M.-C. Hsu, D. Kamensky, S. Stoter, Y. Yu, Y. Zhao, The non-symmetric Nitsche method for the parameter-free imposition of weak boundary and coupling conditions in immersed finite elements, Computer Methods in Applied Mechanics and Engineering 309 (2016) 625-652.

[51] I. Babuška, The finite element method with Lagrangian multipliers, Numerische Mathematik 20 (3) (1973) 179-192.

[52] S. Fernández-Méndez, A. Huerta, Imposing essential boundary conditions in mesh-free methods, Computer Methods in Applied Mechanics and Engineering 193 (12) (2004) 1257-1275.

[53] J. Baiges, R. Codina, F. Henke, S. Shahmiri, W. Wall, A symmetric method for weakly imposing Dirichlet boundary conditions in embedded finite element meshes, International Journal for Numerical Methods in Engineering 90 (5) (2012) 636-658.

[54] K. Höllig, U. Reif, J. Wipper, Weighted extended B-spline approximation of Dirichlet problems, SIAM Journal on Numerical Analysis 39 (2) (2001) 442-462.

[55] K. Höllig, C. Apprich, A. Streit, Introduction to the WEB-method and its applications, Advances in Computational Mathematics 23 (1) (2005) 215-237.

[56] R. Sanches, P. Bornemann, F. Cirak, Immersed b-spline (i-spline) finite element method for geometrically complex domains, Computer Methods in Applied Mechanics and Engineering 200 (13) (2011) 1432-1445.

[57] A. Reusken, Analysis of an extended pressure finite element space for two-phase incompressible flows, Computing and visualization in science 11 (4) (2008) 293-305.

[58] T. Rüberg, F. Cirak, Subdivision-stabilised immersed b-spline finite elements for moving boundary flows, Computer Methods in Applied Mechanics and Engineering 209 (2012) 266-283.

[59] T. Rüberg, F. Cirak, A fixed-grid b-spline finite element technique for fluid-structure interaction, International Journal for Numerical Methods in Fluids 74 (9) (2014) 623-660.

[60] T. Rüberg, F. Cirak, J. García Aznar, An unstructured immersed finite element method for nonlinear solid mechanics, Advanced Modeling and Simulation in Engineering Sciences 3 (1) (2016) 623-660.

61] M. Dauge, A. Düster, E. Rank, Theoretical and Numerical Investigation of the Finite Cell Method, Journal of Scientific Computing 65 (3) (2015) 1039-1064.

[62] R. Becker, M. Braack, A finite element pressure gradient stabilization for the Stokes equations based on local projections, Calcolo 38 (4) (2001) 173-199. 